\title{
COVID año 1. Día Mundial de la Diabetes
}

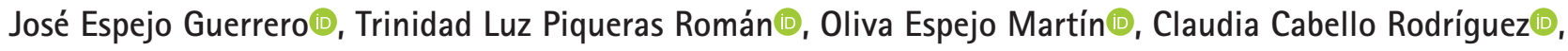 \\ José Espejo Martín(1), Beatriz Delgado Manzano(i) \\ Farmacia José Espejo Guerrero. Adra (Almería).
}

\section{PALABRAS CLAVE}

Diabetes, Glucemia capilar, COVID, Farmacia comunitaria

\section{ABREVIATURAS}

DMD: Día Mundial de la Diabetes IMC: índice de masa corporal iqr: Intervalo intercuartílico

\section{KEYWORDS}

Diabetes, Capillary blood glucose, Covid, Community Pharmacy

\begin{abstract}
RESUMEN
Con motivo de la celebración del Día Mundial de la Diabetes (DMD) en noviembre de 2020, a los usuarios que han acudido a la farmacia se les ha practicado una determinación de glucosa capilar. Los valores hallados se han comparado con la determinación similar que se realizó el mismo DMD en el año 2019 en la misma farmacia, con una muestra diferente. En 2020 se han captado un total de 127 personas frente a las 54 de 2019. La mediana de glucosa no ha variado significativamente de un año a otro ni en el total de pacientes ( $105 \mathrm{mg} / \mathrm{dl}$ en 2020 vs. 105,5 mg/dl en 2019; $p=0,53$ ) ni en los subgrupos de los que toman medicación antidiabética (157 mg/dl en 2020 vs. 187 mg/dl en 2019; p=0,16) ni en los que no toman (103 mg/dl en 2020 vs. 99 mg/dl en 2019; p=0,21). Esta no observación de cambios nos hace reflexionar sobre la forma de detectar futuras alternaciones en el control de la glucosa, sobre todo en pacientes diabéticos. Para ello, será necesario usar estimadores más robustos, como puede ser la hemoglobina glicosilada y trabajar con grupos estables de pacientes.
\end{abstract}

\section{COVID year 1. World Diabetes Day}

\section{ABSTRACT}

On the occasion of the celebration of World Diabetes Day (WDD) in 2020, users who have come to the Pharmacy have been given a determination of capillary glucose. Compared to the similar determination made by the same WDD in 2019 at the same Pharmacy, the values found have been compared with a different sample. In 2020, a total of 127 people were recruited compared to 54 in 2019. Median glucose has not changed significantly from year to year or in total patients $(105 \mathrm{mg} / \mathrm{dL}$ in $2020 \mathrm{vs} 105.5 \mathrm{mg} / \mathrm{dL}$ in 2019; $p=0.53$ ) neither in the subgroups of those taking antidiabetic medication ( $157 \mathrm{mg} / \mathrm{dL}$ in 2020 vs $187 \mathrm{mg} / \mathrm{dL}$ in 2019; $\mathrm{p=0.16}$ ) or in those who do not take (103 mg/dl in $2020 \mathrm{vs} 99 \mathrm{mg} / \mathrm{dL}$ in 2019; $\mathrm{p}=0.21$ ). This non-observation of changes makes us reflect on how to detect future glucose control alternations, especially in diabetic patients. To do this, it will be necessary to use more robust estimators, such as glycosylated hemoglobin and work with stable groups of patients.

\section{INTRODUCCIÓN}

Cada 14 de noviembre se celebra el Día Mundial de la Diabetes (DMD). Aprovechando esta celebración, algunas farmacias realizan pruebas de cribado de la diabetes a sus usuarios $(1,2,3)$. Este cribado suele incluir, entre otras, la determinación de la glucemia capilar. El pasado 2019 hemos celebrado tal fecha conforme a lo expuesto anteriormente. En el 2020 lo hemos repetido con un grupo de personas diferente al de 2019.
En el año 2020 nos ha sobrevenido la pandemia por el coronavirus SARS-CoV-2. El desplazamiento de la atención sanitaria del sistema público de salud a los pacientes con la COVID-19 ha trastocado los planes de salud en los pacientes no COVID, lo que puede repercutir negativamente en el buen control de enfermedades crónicas $(4,5,6)$. En este contexto, el objetivo del presente trabajo es comparar los resultados de las glucemias observadas en 2020 con las de 2019, en los respectivos DMD en nuestra farmacia.
Recibido: 14/12/2020

Aceptado: 03/05/2021 Disponible online: $02 / 07 / 2021$
Financiación: ninguna.

Conflicto de intereses: ninguno.

Cite este artículo como: Espejo J, Piqueras TL, Espejo O, Cabello C, Espejo J, Delgado B. COVID año 1. Día Mundial de la Diabetes. Farmacéuticos Comunitarios. 2021 Jul 02; 13 (3): 25-28. doi:10.33620/FC.2173-9218.(2021/Nol13).003.04 Correspondencia: Pepe Espejo (jespejo18@gmail.com).

ISSN 1885-8619 @SEFAC (Sociedad Española de Farmacia Clínica, Familiar y Comunitaria). Todos los derechos reservados. 


\section{MATERIAL Y MÉTODOS}

El día 17 de noviembre de 2020 a toda persona mayor de edad que ha visitado nuestra farmacia se le ha invitado a la determinación de glucosa capilar. Además, se han registrado las siguientes variables: edad, sexo, horas de ayuno, toma de medicación antidiabética, peso, talla y cálculo del índice de masa corporal (IMC). Según los resultados obtenidos se ha aconsejado al usuario lo que debería hacer para mantener 0 mejorar en su caso los niveles de glucemia, lo cual incluye la derivación a su médico de cabecera cuando así se ha creído necesario. A los que presentaban una glucosa superior a $120 \mathrm{mg} / \mathrm{dl}$ y no estaban en ayunas se les ha citado al dia siguiente en ayunas para repetir la determinación.

Los valores de glucemia hallados en los individuos el día 17 se han comparado con las del grupo formado por los individuos que visitaron la farmacia el 14 de noviembre de 2019 y a los que se les practicó igualmente una determinación de glucosa capilar con motivo del DMD.

Para el análisis de los datos se ha realizado una descripción comparativa de los años 2019 y 2020 en las variables recogidas, las cuantitativas con la mediana y rango intercuartílico, debido a la falta de normalidad en sus distribuciones (prueba de Saphiro-Wilk significativa) y las cualitativas con porcentaje. Para comparar los niveles de glucosa de ambos años, dada la distribución no normal de la variable, se ha practicado la prueba no paramétrica de Fisher-Pitman (7), con el cálculo del intervalo de confianza de la diferencia de medianas (8). A continuación se ha practicado una regresión lineal múltiple, siendo la variable resultado la glucemia capilar y como variables explicativas el año, la edad, el sexo, la toma de medicación antidiabética y el índice de masa corporal.

\section{RESULTADOS}

En el año 2020 se han captado un total de 127 personas, frente a las 54 de 2019 (tabla 1). Las medianas de edad
Tabla 2 Valores de glucosa capilar por años y por grupos de personas

\begin{tabular}{|l|c|c|c|c|c|}
\hline & Año 2020 & \multicolumn{2}{|c|}{ Año 2019 } & \\
\hline$n$ & \multicolumn{2}{|c|}{127} & \multicolumn{2}{|c|}{54} & \\
\hline $\begin{array}{l}\text { Glucosa (mg/dl) (mediana, } \\
\text { iqr) }\end{array}$ & 105 & 32 & 105,5 & 33 & NS \\
\hline
\end{tabular}

\begin{tabular}{|l|c|c|c|c|c|}
\hline Glucosa por grupos \\
\hline $\begin{array}{l}\text { Entre los que no toman } \\
\text { medicación antidiabética } \\
\text { (mediana, iqr) }\end{array}$ & 103 & 21 & 99 & 25 & NS \\
\hline $\mathrm{n}$ & \multicolumn{2}{|c|}{99} & \multicolumn{2}{|c|}{41} & \\
\hline $\begin{array}{l}\text { Entre los que si toman } \\
\text { medicación antidiabética } \\
\text { (mediana, iqr) }\end{array}$ & 157 & 86 & 187 & 125 & NS \\
\hline $\mathrm{n}$ & 28 & \multicolumn{2}{|c|}{13} & \\
\hline
\end{tabular}

iqr: rango intercuartílico; NS: no significativo.

fueron de 59 y 62 años respectivamente, con un 70,9\% de mujeres en 2020 vs. 77,8\% en 2019. Tomaban medicación antidiabética el 22,1\% en un caso y 24,1\% en 2019.

Los valores de glucosa (tabla 2) en mediana son de $105 \mathrm{mg} /$ dl en 2020 frente a 105,5 mg/dl en 2019, diferencias que no son estadísticamente significativas ni en el total de pacientes (IC 95\%: -11 a 6; $p=0,53$ ) ni en el grupo de tratamiento con antidiabéticos (157 mg/dl en 2020 vs. $187 \mathrm{mg} / \mathrm{dl}$ en 2019; IC95\%: -28 a 82; $p=0,16)$ ni entre los que no toman (103 mg/dl vs. $99 \mathrm{mg} / \mathrm{dl}$ en 2019; IC95\%: -11 a 3; $p=0,21$ ) (figuras 1 y 2).

Se ha realizado una regresión lineal múltiple para aislar el posible efecto del año, ajustando por las demás variables. No se observa un valor estadísticamente significativo en la diferencia de año $(b=-4,19 ; p=0,44)$, siendo las únicas variables con significación estadística, la edad $(b=0,35$; $p=0,033)$ la toma de medicación $(b=58,18 ; p<0,001)$ y las horas de ayuno $(b=-2,76 ; p=0,003)$.

Tabla 1 Descripción de las muestras observadas

\begin{tabular}{|c|c|c|c|c|c|}
\hline & \multicolumn{2}{|c|}{ Año 2020} & \multicolumn{2}{|c|}{ Año 2019} & $\mathrm{p}$ \\
\hline$n$ & \multicolumn{2}{|c|}{127} & \multicolumn{2}{|c|}{54} & \\
\hline Edad (mediana, iqr) & 59 & 26 & 62 & 19 & NS \\
\hline Sexo (mujer $n, \%)$ & 90 & 70,9 & 42 & 77,8 & NS \\
\hline Horas de ayuno (mediana, iqr) & 2,5 & 1,75 & 2,5 & 2 & NS \\
\hline Toma de medicación antidiabética $(n, \%)$ & 28 & 22,1 & 13 & 24,1 & NS \\
\hline Peso (kg) (mediana, iqr) & 74,2 & 22,4 & 70,75 & 22,6 & NS \\
\hline Talla (cm) (mediana, iqr) & 161 & 11 & 163 & 12 & NS \\
\hline IMC (kg/m²) (mediana, iqr) & 28,02 & 6,82 & 27,10 & 7,56 & NS \\
\hline
\end{tabular}

iqr: rango intercuartílico; IMC: indice de masa corporal; NS: no significativo. 


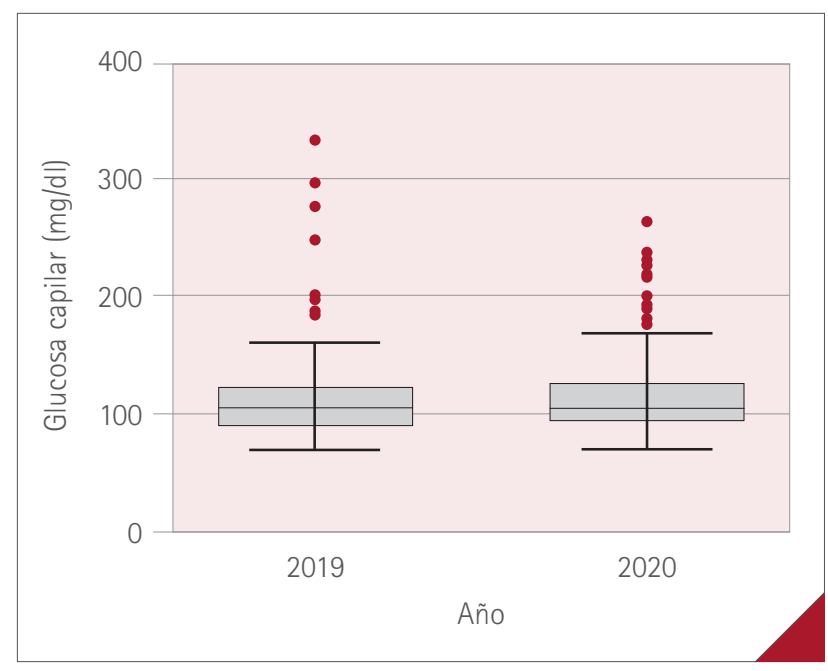

Figura 1 Gráfico de caja de valores de glucosa por año

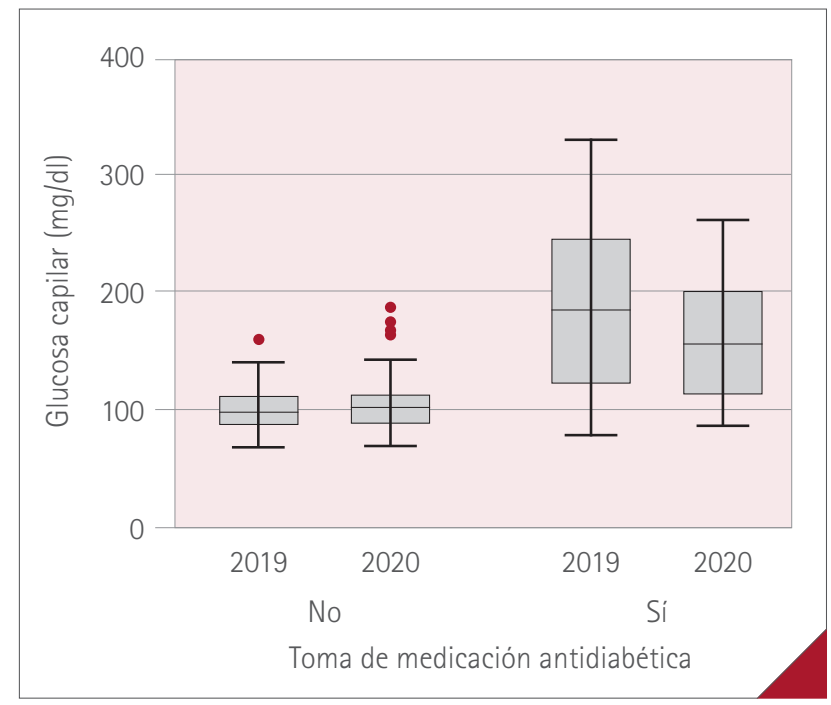

Figura 2 Gráfico de caja de niveles de glucosa por toma de medicación y año

\section{DISCUSIÓN}

En el año 2020 se han captado 127 personas, más del doble que en el 2019, en que fueron 54. Ello creemos que se debe fundamentalmente al procedimiento de captación, que se ha producido a la entrada en la farmacia, que no a la salida como el año anterior, y a la existencia de una persona dedicada exclusivamente a dicho proceso de captación.

Aun siendo grupos no equivalente de comparación, son similares en cuanto a la estructura de variables registradas (tabla 1). Los valores de glucosa en mediana no varían de un año a otro ni en el total de personas (105 en 2020 vs. 105,5 en 2019) ni en los que están o no con tratamiento antidiabético (tabla 2). Lo que en un principio se podría hipotetizar sobre la influencia de este tiempo de convivencia con el coronavirus, como podría ser un incremento de la media de glucosa en la muestra de 2020, no ha ocurrido. En una segunda aproximación se podría hipotetizar que realmente a quien debería afectar no es a la población general, sino a los diabéticos (en nuestro caso los que toman medicación antidiabética), pues al estar supuestamente menos controlados, en teoría deberían presentar valores medios de glucosa más elevados, lo cual tampoco ocurre (157 en 2020 vs. 187 mg/dl en 2019).

Pero si profundizamos en nuestro razonamiento, podemos inducir que los cambios de glucosa, a nivel poblacional, no pueden ser de un año para otro clínicamente relevantes. $Y$, si es que los diabéticos estuvieran menos controlados (lo desconocemos), lo que en un primer momento se observaría sería una elevación de un parámetro más robusto en cuanto a la evaluación del buen o mal control, cual es la hemoglobina glicosilada y no así de una glucemia al azar que, como su propio nombre indica, está influenciada por la mejor o peor suerte del día y hora en que se realiza. Y, en un segundo estadio, habría que observar la posible afectación de los órganos diana debido a las complicaciones micro y macrovasculares de la diabetes, lo que quedaría reflejado con un incremento en la incidencia de las mismas.

Resultados en el mismo sentido, no cambio e incluso mejora, se han obtenido en otros estudios $(9,10)$, aunque hay diseñados ecuaciones para predicción del aumento de la hemoglobina glicosilada en periodos de catástrofes nacionales (11), ecuaciones que en este caso no han funcionado (9).

Por tanto, creemos que para analizar la repercusión de este "periodo COVID" en las enfermedades crónicas no COVID, como puede ser la diabetes, es necesario trabajar con grupos estables y con marcadores más robustos que la glucosa capilar. Y para observar las diferencias a nivel poblacional habremos de recurrir a las estadísticas de salud en los años venideros.

\section{CONCLUSIONES}

Los valores de glucemia capilar en los usuarios analizados el DMD en 2020 no son estadísticamente diferentes a los analizados en los usuarios del DMD en 2019.

Esta no diferencia se observa tanto en el total de individuos como en los grupos de los que toman medicación antidiabética y el grupo de los que no toman, analizados por separado.

Para futuras investigaciones habrá que usar parámetros más estables que la glucosa al azar y hacer un seguimiento a grupos cerrados de pacientes.

A largo plazo habrá que seguir la evolución de la incidencia de las complicaciones micro y macrovasculares de la diabetes. 


\section{AGRADECIMIENTOS}

Agradecemos a la Sta. Doña Laura Vela Parrilla, alumna en prácticas de Técnica en Farmacia, su participación en el proceso de captación de usuarios en 2020. Agradecemos a Acofarma su colaboración en ambos años en la prestación de material, aparatos y tiras reactivas.

\section{REFERENCIAS BIBLIOGRÁFICAS}

1. Abalde P, Touriño E Gómez P Rodríguez EM. Resultados de un cribado de diabetes realizado en una farmacia comunitaria. Farmacéuticos Comunitarios 2014; 6 (Suplemento 1). Disponible en: https:// www.farmaceuticoscomunitarios.org/es/journal-article/resultados-cribado-diabetes-realizado-una-farmacia-comunitaria

2. Ramos $R$, Romero J, Relinque $V$, Naranjo $M A$, Navarro R. Campaña de identificación de pacientes con riesgo elevado de padecer diabetes en farmacias comunitarias de Málaga. Farmacéuticos Comunitarios 2014; 6 (Suplemento 1). Disponible en: https://www.farmaceuticoscomunitarios.org/es/journal-article/campana-identificacion-pacientes-con-riesgo-elevado-padecer-diabetes-farmacias

3. Barreiro M, Mahiques MJ, Serrano $N$, Andrés $C$, Iracheta $M$, Baixauli VJ, Benet $A$, Andrés N, Fornos JA. Proyecto 'Campaña Día Mundial de la Diabetes en farmacias comunitarias': detección de personas en riesgo de padecer diabetes en 2014, 2016 y 2017 en farmacias comunitarias españolas. Farmacéuticos Comunitarios 2018; 10 (Suplemento 1): 38. Disponible en: https://www.farmaceuticoscomunitarios.org/es/journal-article/proyecto-campana-dia-mundial-diabetes-farmacias-comunitarias-deteccion-personas-0

4. Francisco Cañizares. Controlar la diabetes para proteger el corazón, un desafio en medio de la pandemia. El País. 4/12/2020; Sociedad. Disponible en: https://elpais.com/sociedad/mas-corazon-menos-diabetes-2/
5. de Nicolás Jiménez JM, Blázquez Recio LM, Fabregat Domínguez MT, Palomo Cobos L. COVID-19 y esfuerzo asistencial en atención primaria [COVID-19 and assistance effort in Primary Care]. Aten Primaria. 2020;52(8):588-590. doi:10.1016/j.aprim.2020.06.002

6. Carlos Miranda Fernández-Santos, María Eva Sáez Torralba, Francisco José Sáez Martínez. La cronicidad en los tiempos del cólera o la diabetes como ejemplo de lo que no hay que hacer: diabetes y covid-19. Med Gen Fam v9n3, Revisión. Disponible en: http://mgyf. org/wp-content/uploads/2020/07/MGYF2020_034.pdf

7. Kaiser J. An Exact and a Monte Carlo Proposal to the Fisher-Pitman Permutation Tests for Paired Replicates and for Independent Samples. The Stata Journal. 2007; 7 (3): 402-412. doi:10.1177/ $1536867 \times 0700700307$

8. Newson, R. 2000b. snp16: Robust confidence intervals for median and other percentile differences between groups, Stata Technical Bulletin 58: 30-35. Reprinted in Stata Technical Bulletin Reprints, vol. 10, pp. 324-331. College Station, TX: Stata Press. Disponible en: https://www.stata-journal.com/article.html?article $=$ st0007

9. Rastogi A, Hiteshi P, Bhansali A. Improved glycemic control amongst people with long-standing diabetes during COVID-19 lockdown: a prospective, observational, nested cohort study. Int J Diabetes Dev Ctries. 2020 Oct 21:1-6. doi:10.1007/s13410-020-00880-X

10. Maddaloni E, Coraggio L, Pieralice S, Carlone A, Pozzilli P, Buzzetti R. Effects of COVID-19 Lockdown on Glucose Control: Continuous Glucose Monitoring Data From People With Diabetes on Intensive Insulin Therapy. Diabetes Care. 2020 Aug;43(8):e86-e87. doi:10.2337| dc20-0954

11. Ghosal $S$, Sinha B, Majumder M, Misra A. Estimation of effects of nationwide lockdown for containing coronavirus infection on worsening of glycosylated haemoglobin and increase in diabetes-related complications: A simulation model using multivariate regression analysis. Diabetes Metab Syndr. 2020 Jul-Aug;14(4):319-323. doi:10.1016/j.dsx.2020.03.014 